\title{
FORNECIMENTO DE HORTIFRUTIGRANJEIROS PARA UNIDADES DE ALIMENTAÇÃO E NUTRIÇÃO HOSPITALARES ${ }^{1}$
}

\author{
Aline Petter SCHNEIDER ${ }^{2 * *}$
}

\section{RESUMO}

O fornecimento de hortifrutigranjeiros para o setor hospitalar é um importante segmento dentro da cadeia de suprimento agroalimentar. Este fornecimento demanda altos padrões de qualidade, incluindo o cumprimento de normas higiênico-sanitárias, padrão do tamanho, da forma, da procedência, da isenção de resíduos e agrotóxicos, entre outros. O objetivo deste estudo foi analisar o processo atual de fornecimento de hortifrutigranjeiros e identificar as necessidades e exigências das unidades hospitalares. A metodologia empregada foi a de estudo de múltiplos casos. Foram analisados os hospitais do município de Porto Alegre (Rio Grande do Sul, Brasil) e a Central de Abastecimento e Distribuição de Hortifrutigranjeiros e seus distribuidores. Os resultados obtidos demonstram, por parte dos hospitais, grande preocupação quanto ao atual método de fornecimento, bem como a necessidade de um fornecimento especializado. No que se refere aos fornecedores, verificou-se o descumprimento de fatores considerados indispensáveis pelos hospitais, entre os quais o controle na utilização de agrotóxicos, as condições higiênico-sanitárias, a procedência e as condições de transporte. Como conclusão, verificou-se que o fornecimento atual não é eficiente e se dá de maneira inadequada, não atendendo a maioria dos requisitos hospitalares de fornecimento. Sugere-se a implementação da metodologia de Análise dos Perigos e Pontos Críticos de Controle, das Boas Práticas de Fabricação, do Gerenciamento da Cadeia de Suprimentos e do Cooperativismo, como propostas para a otimização do fornecimento.

Palavras-chave: unidades hospitalares, serviço hospitalar de nutrição, administração de materiais.

\section{SUMMARY}

THE SUPPLY OF FRUITS AND VEGETABLES FOR FOOD AND NUTRITION HOSPITALS UNITS. The food and vegetables supply to hospitals is an important part of food supply channel. This supply demands, among others, high patterns of quality, including size, shape, origin, low levels of residuals and pesticides, as well as the accomplishment of hygiene and sanitary rules. The aim of this study was to evaluate the current process of food and vegetables supply for the hospitals and to verify the efficiency of such process, according to the needs and requirements of hospital units. The methodology was a case-series study. The hospital situated in the City of Porto Alegre, Rio Grande do Sul, Brazil and a central of store and distribution of food and vegetables, as well as its suppliers were analysed. The results demonstrate that the hospitals have a great concern regarding the current supply method and, in addition, that they prefer a specialized one. In reference to suppliers, it was verified the non-observance of factors considered as indispensable by hospitals. These include, for instance, the control for use of pesticides, the hygiene and sanitary conditions, the origin and the transportation. In conclusion, it was verified that the current supply is not efficient, that it occurs in an inappropriate way and that it does not assist the most of the hospital supply requirements. It is suggested the implementation of the hazard analysis and critical control points, the good practice of manufacturing, the supply chain management, and a cooperative approach to optimize the food and vegetable supply.

Keywords: hospital units, nutritional hospital service, management materials.

\section{1 - INTRODUÇÃO}

As Unidades de Alimentação e Nutrição (UANs) constituem importantes setores dentro das empresas onde estão estabelecidas. Mais ainda, em se tratando de hospitais, onde o fornecimento de alimentos destina-se ao preparo de dietas especializadas que visam à recuperação e tratamento dos pacientes. Dentre os objetivos das UANs em uma organização hospitalar, verifica-se como prioridade o fornecimento de alimentação adequada, balanceada e segura.

Para o cumprimento deste importante requisito, é indispensável que o fornecimento de hortifrutigranjeiros para

${ }^{1}$ Recebido para publicação em 15/03/2004. Aceito para publicação em 28/04/2006 (001309)

${ }^{2} \mathrm{Nutricionista,} \mathrm{especialista} \mathrm{em} \mathrm{Administração} \mathrm{Hospitalar} \mathrm{e} \mathrm{mestre} \mathrm{em} \mathrm{Agro-}$ negócios pela Universidade Federal do Rio Grande do Sul. Doutoranda em Ciências da Saúde pela Pontifícia Universidade Católica do Rio Grande do Sul. Docente do Centro Universitário Univates - Faculdade de Nutrição e de Enfermagem e docente da Rede Metodista de Educação (IPA) - Faculdade de Nutrição e de Administração Hospitalar.

Rua Professor Ulisses Cabral, 1.310/304

CEP 91330-520, Porto Alegre (RS)

E-mail:alinepst@terra.com.br

*A quem a correspondência deve ser enviada os hospitais atenda as necessidades higiênico-sanitárias e, mais do que isso, garanta a uniformidade dos produtos quanto a tamanho e forma, bem como a integridade de suas características organolépticas.

O segmento hospitalar representa um dos importantes setores de recepção de produtos, ao qual é destinada a distribuição de hortifrutigranjeiros, elo final da cadeia produtiva. A cadeia de suprimentos pode ser definida como uma rede de empresas que obtém matérias-primas, as processa em produtos, primeiramente intermediários e, em seguida, em produtos acabados, distribuindo-os aos consumidores finais [14].

COOPER E ELLRAM [8] apontam importantes razões para a formação de cadeias: a) redução dos investimentos em estoques ao longo da cadeia total; b) melhora no serviço prestado ao cliente; e c) ajuda na construção de uma vantagem competitiva para o setor como um todo.

Mas se alguns autores apontam vantagens na formação de cadeias, outros alertam para seus problemas. Para Davis [10], a falta de comunicação e o desconhecimento sobre as necessidades de cada elo da cadeia é a real dificuldade das cadeias de suprimentos. LEE E BILLINGTON 
[15] apontam outros perigos, como a falta de definição das informações que devem circular entre as empresas e problemas operacionais e estratégicos. Para a eficiência e eficácia do processo, é indispensável a existência de um fluxo coerente de informação ao longo da cadeia [22].

A discussão sobre Gerenciamento da Cadeia de Suprimentos iniciou-se no contexto logístico de gerenciamento de estoque. A idéia era reduzir os estoques dos membros pertencentes à cadeia e, conseqüentemente, o custo total da cadeia [22].

O gerenciamento logístico está primeiramente preocupado com a otimização de fluxos dentro da organização, enquanto o gerenciamento da cadeia de suprimentos reconhece que a integração interna não é suficiente, ou seja, ela vê a cadeia de suprimentos como uma entidade única que trata do transporte e estocagem de bens e do fluxo de informações associado aos mesmos, mas isto, do produtor de matéria-prima até o consumidor final, na cadeia como um todo [7].

Observam-se três pontos-chave para o estabelecimento do gerenciamento da cadeia: conectividade entre os membros, alinhamento dos sistemas de suporte interorganizacionais e compartilhamento de recursos - informações e expertises [15].

Cadeia ou filière agroalimentar é uma seqüência de operações que transforma uma commodity em um produto pronto para o consumidor final [23]. Esta cadeia é composta de elos que vão desde a matéria-prima, passando pela agricultura, indústria, distribuição (atacado e varejo) até chegar ao consumidor final.

Relatos da Organização Mundial da Saúde (OMS) e informações recentes sobre doenças de origem alimentar no Brasil, mostram que mais de $60 \%$ são toxiinfecções alimentares. Isto se deve às práticas inadequadas de manipulação, matérias-primas contaminadas, falta de higiene ao longo da cadeia, desde a produção até o consumo, além de equipamentos e estrutura operacional deficientes e, principalmente, inadequação envolvendo o controle de tempo e temperatura [13].

A Organização Mundial do Comércio (OMC) reconhece a Comissão do Codex Alimentarius como organismo internacional competente em matéria de normalização da inocuidade dos alimentos [13]. No Brasil, o esforço de adequação nacional às normas do Codex Alimentarius vem sendo conduzido de forma conjunta entre o Ministério da Agricultura e do Abastecimento, Ministério da Saúde (por meio da Agência Nacional de Vigilância Sanitária - Anvisa) e Ministério do Desenvolvimento, Indústria e Comércio Exterior (por meio do Instituto Nacional de Metrologia, Normalização e Qualidade Industrial - Inmetro), de acordo com os comitês específicos.

As Boas Práticas de Fabricação (BPF) são um conjunto de normas empregadas em produtos, processos, serviços e edificações, visando a promoção e a certificação da qualidade e da segurança do alimento. No Brasil, as BPF são legalmente regidas pelas Portarias 1.428/93-MS [3] e 326/97-SVS/MS [4].

A qualidade da matéria-prima, a arquitetura dos equipamentos e das instalações, as condições higiênicas do ambiente de trabalho, as técnicas de manipulação dos alimentos e a saúde dos funcionários são fatores importantes a serem considerados na produção de alimentos seguros e de qualidade, devendo, portanto, ser levados em conta nas BPF.

A avaliação dessas BPF em estabelecimentos de produção ou de comercialização de alimentos, por meio de utilização de questionários apropriados, é citada como subsídio para qualificação e triagem de fornecedores [2, 19], como base para vistoria fiscal sanitária [5, 18, 21], para a verificação, pelo próprio estabelecimento, do cumprimento das BPF [6, 20], ou como base para a implantação do sistema Análise de Perigos e Pontos Críticos de Controle (APPCC) [1, 16].

Sabe-se, todavia, que inexiste qualquer diferenciação de hortifrutigranjeiros, seja do ponto de vista produtivo, seja do ponto de vista logístico, para atendimento da demanda hospitalar, e que o fornecimento se dá a partir de distribuidores provenientes de grandes centrais de abastecimento e distribuição (Ceasas), as quais recebem seus produtos dos mais diversos produtores.

Há, ainda, amplo desconhecimento sobre a existência de controle na utilização de agrotóxicos. Tendo em vista as considerações feitas acima, o objetivo deste estudo foi descrever e analisar o processo atual de fornecimento de produtos hortifrutigranjeiros e identificar as necessidades e exigências de qualidade para as unidades hospitalares.

\section{2 - MATERIAL E MÉTODOS}

O estudo foi realizado na cidade de Porto Alegre (RS), entre outubro de 2002 e março de 2003. Participaram da pesquisa os hospitais e os fornecedores de hortifrutigranjeiros.

Para atingir o objetivo deste estudo foi realizada uma pesquisa observacional, de caráter transversal, que consistiu de um estudo de casos múltiplos. A primeira fase da coleta de dados foi feita por meio de entrevista semi-estruturada por questionário com as nutricionistas dos hospitais, seguida de entrevista e visita técnica com os fornecedores.

O município de Porto Alegre possui 36 hospitais. Destes, três não participaram da pesquisa por não demonstrar interesse ou pela ausência de nutricionista na ocasião da pesquisa. Os fornecedores de hortifrutigranjeiros para os hospitais de Porto Alegre foram identificados pela entrevista realizada nos hospitais com as nutricionistas de produção ou supervisoras do serviço. Fizeram parte da amostra os fornecedores que atendem dois ou mais hospitais e 250 leitos ou mais na cidade de Porto Alegre.

Os questionários e o roteiro da visita técnica foram construídos particularmente para atender os objetivos deste estudo, com base nas normativas, e elaborados com base em 
critérios propostos pela literatura e legislações vigentes [ 1,2 , $3,4,5,6,16,18,19,20]$. O questionário foi previamente testado em uma amostra não incluída nesta pesquisa. A entrevista teve como objetivo determinar as necessidades e as exigências de qualidade quanto aos hortifrutigranjeiros fornecidos aos hospitais. As entrevistas com as nutricionistas ocorreram pessoalmente ou por telefone.

Os itens dos questionários foram agrupados, por assunto, em 14 questões conforme é apresentado na Tabela 1.

TABELA 1 - Perguntas aplicadas aos hospitais

\begin{tabular}{|c|c|}
\hline Pergunta & $\begin{array}{l}\text { Número da } \\
\text { Pergunta }\end{array}$ \\
\hline $\begin{array}{l}\text { O padrão do tamanho das frutas é um requisito importante } \\
\text { de fornecimento }\end{array}$ & 1 \\
\hline $\begin{array}{l}\text { O padrão do tamanho das hortaliças é um requisito } \\
\text { importante de fornecimento }\end{array}$ & 2 \\
\hline $\begin{array}{l}\text { As condições de forma e formato dos hortifrutigranjeiros } \\
\text { são avaliadas no fornecimento }\end{array}$ & 3 \\
\hline $\begin{array}{l}\text { Considero importante a cor dos hortifrutigranjeiros no seu } \\
\text { fornecimento }\end{array}$ & 4 \\
\hline $\begin{array}{l}\text { É indispensável que haja controle na utilização de } \\
\text { agrotóxicos nos hortifrutigranjeiros }\end{array}$ & 5 \\
\hline $\begin{array}{l}\text { É necessário que os hortifrutigranjeiros sejam isentos de } \\
\text { resíduos químicos }\end{array}$ & 6 \\
\hline $\begin{array}{l}\text { Considero importante a qualidade intrínseca dos produtos } \\
\text { hortifrutigranjeiros }\end{array}$ & 7 \\
\hline $\begin{array}{l}\text { Costuma realizar visitas antes de iniciar com um novo } \\
\text { fornecedor }\end{array}$ & 8 \\
\hline Obtenho informações sobre a proveniência dos produtos & 9 \\
\hline $\begin{array}{l}\text { É realizado o controle dos fatores de correção para } \\
\text { verificar se as sobras estão de acordo com as indicações } \\
\text { encontradas na literatura especializada }\end{array}$ & 10 \\
\hline O hospital costuma devolver mercadorias ao fornecedor & 11 \\
\hline $\begin{array}{l}\text { É realizada análise química e microbiológica periódica nos } \\
\text { hortifrutigranjeiros }\end{array}$ & 12 \\
\hline $\begin{array}{l}\text { Acha possível pagar mais por hortifrutigranjeiros } \\
\text { produzidos ou selecionados para os hospitais }\end{array}$ & 13 \\
\hline $\begin{array}{l}\text { Está satisfeito com o fornecimento atual de } \\
\text { hortifrutigranjeiros }\end{array}$ & 14 \\
\hline
\end{tabular}

Fonte: Dados da Pesquisa

Em um segundo momento realizou-se a avaliação dos fornecedores. Foram selecionados os fornecedores que atendiam dois ou mais hospitais e no mínimo 250 leitos hospitalares. Na ausência do responsável, foi entrevistado o distribuidor ou o entregador. Realizaram-se visitas técnicas para observação direta das variáveis em estudo. Os processos avaliados foram: de seleção, de armazenamento e de distribuição dos hortifrutigranjeiros.

A análise estatística foi realizada por meio do Programa SPSS, usando as tabelas com freqüências absolutas e percentuais, de acordo com os resultados obtidos.
Foi usado o teste $\mathrm{x}^{2}$ de Mantel-Haenzsel para tendência linear e odds ratio no intervalo de confiança de $95 \%$, para avaliar o relacionamento entre os grupos.

\section{3 - RESULTADOS E DISCUSSÃO}

O município de Porto Alegre possui 36 hospitais. Destes, três não participaram da pesquisa por não demonstrar interesse, ou pela ausência de nutricionista na ocasião da pesquisa.

O Complexo Hospitalar Santa Casa de Misericórdia é composto de seis hospitais e para os quais existem três serviços de produção de alimentos, sendo que o Hospital Santa Rita e o Hospital Santo Antônio da Criança têm esta divisão junto ao hospital. Os quatro hospitais restantes são atendidos pela mesma unidade de produção. Os demais hospitais [26] têm, cada qual, suas unidades de produção de alimentos independentes.

Durante as entrevistas, verificou-se junto às nutricionistas que atuam em hospitais onde o processo de compra se dá por licitação, alguns problemas, principalmente pelo prazo de duração do contrato, de três meses até um ano, e da dificuldade de suspensão do mesmo. Foi referido ainda pelas nutricionistas destes hospitais que o manejo com os fornecedores é difícil, não havendo interesse especial em atender os requisitos destes hospitais. Dessa forma, a análise dos dados foi dividida em duas partes: os hospitais que compram pelo processo de licitação e os que não compram pelo processo de licitação.

Os fornecedores de hortifrutigranjeiros para os hospitais de Porto Alegre foram identificados pela entrevista realizada nos hospitais com as nutricionistas de produção ou supervisoras do serviço. O nome dos fornecedores não será divulgado e, por isso, foram denominados por letras, conforme estão apresentados na Tabela 2.

TABELA 2 - Levantamento de fornecedores

\begin{tabular}{lcc}
\hline Fornecedores & $\begin{array}{c}\mathbf{N}^{\circ} \text { de leitos } \\
\text { Atendidos }\end{array}$ & $\begin{array}{c}\mathbf{N}^{\circ} \text { de } \\
\text { hospitais }\end{array}$ \\
\hline $\mathrm{A}$ & $2.290(29,40 \%)$ & $4(14,28 \%)$ \\
$\mathrm{B}$ & $1.370(17,58 \%)$ & $3(10,71 \%)$ \\
$\mathrm{C}$ & $1.200(15,40 \%)$ & $5(17,85 \%)$ \\
$\mathrm{D}$ & $700(8,98 \%)$ & $2(7,14 \%)$ \\
$\mathrm{E}$ & $610(7,83 \%)$ & $4(14,28 \%)$ \\
$\mathrm{F}$ & $560(7,19 \%)$ & $1(3,57 \%)$ \\
G & $380(4,88 \%)$ & $3(10,71 \%)$ \\
$\mathrm{H}$ & $260(3,33 \%)$ & $2(7,14 \%)$ \\
I & $130(1,66 \%)$ & $1(3,57 \%)$ \\
J & $120(1,54 \%)$ & $1(3,57 \%)$ \\
K & $74(0,95 \%)$ & $1(3,57 \%)$ \\
L & $60(0,77 \%)$ & $1(3,57 \%)$ \\
M & $35(0,45 \%)$ & $1(3,57 \%)$ \\
\hline Total & Total leitos: 7.789 & Total de hospitais: 28 \\
fornecedores: $\mathbf{1 3}$ & $(\mathbf{1 0 0 \% )}$ & $(\mathbf{1 0 0 \% )}$ \\
\hline Fonte: Dados da pesquisa & &
\end{tabular}

Do total da amostra (33 hospitais), quatro hospitais realizam as compras diretamente na Ceasa. Apenas um 
hospital tem horta própria e complementa seu pedido com compras na Ceasa.

Nesta pesquisa, foram visitados os oito principais fornecedores, levando-se em conta a relevância em termos de volume de pedido $(94,64 \%$ dos leitos hospitalares) e número de hospitais atendidos (82,15\% dos hospitais).

As nutricionistas dos hospitais foram entrevistadas através de perguntas semi-estruturadas. As análises dos dados encontram-se nas Tabelas 3 e 4.

Com relação à escolha do fornecedor pelos hospitais que compram sem licitação, na maior parte das vezes, não são realizadas visitas anteriores ao fechamento do contrato. Pelo mesmo grupo, foi referido pelos entrevistados que os aspectos mais importantes são: 1) higiene do produto, das caixas e do caminhão; 2) disponibilidade em atender pedidos fora do planejamento; 3) manutenção do padrão; e 4) confiança no fornecedor.

Com relação à escolha do fornecedor pelos hospitais que compram por licitação, foi dito pelos entrevistados que os aspectos mais importantes são: 1) disponibilidade em atender pedidos fora do planejamento; 2) higiene do produto, das caixas e do caminhão; 3) segurança dos produtos; e 4) confiança no fornecedor.

Foram observadas algumas diferenças que não foram estatisticamente significativas, entre os dois grupos analisados: os que compram e os que não compram pelo processo de licitação. O grupo que compra sem processo de licitação obtém mais informações sobre a proveniência dos produtos do que os que compram pelo processo de licitação. Em contrapartida, os hospitais que adquirem produtos sem processo de licitação realizam menos visitas técnicas que os hospitais que compram por licitação.

Os hospitais que compram por licitação fazem maior controle dos fatores de correção do que os hospitais que não utilizam este processo ( $p<0,001$ ). Corresponde a 54,54\% dos hospitais que compram pelo processo de licitação e a $15,78 \%$ dos que não usam de licitação.

Apesar das dificuldades relatadas pelas nutricionistas dos hospitais que compram pelo processo de licitação, no início das entrevistas, o grau de satisfação desses hospitais (54,54\%) é semelhante quando comparado ao dos hospitais que não compram por licitação $(57,88 \%)$, não tendo sido encontrada significância estatística.

TABELA 3 - Análise dos critérios e ações do fornecimento de hortifrutigranjeiros (sem processo de licitação de compras)

\begin{tabular}{|c|c|c|c|c|c|}
\hline Número da Pergunta & $\begin{array}{l}\text { Discorda } \\
\text { totalmente }\end{array}$ & Discorda & $\begin{array}{c}\text { Imparcial/ } \\
\text { Neutro }\end{array}$ & Concorda & Concorda totalmente \\
\hline 1 & $3(15,78 \%)$ & Zero & Zero & $3(15,78 \%)$ & $13(68,42 \%)$ \\
\hline 2 & $2(10,52 \%)$ & $2(10,52 \%)$ & $6(31,57 \%)$ & $2(10,52 \%)$ & $7(36,84 \%)$ \\
\hline 3 & $1(5,26 \%)$ & Zero & Zero & $3(15,78 \%)$ & $15(78,94 \%)$ \\
\hline 4 & $1(5,26 \%)$ & Zero & Zero & $1(5,26 \%)$ & $17(89,47 \%)$ \\
\hline 5 & Zero & Zero & $1(5,26 \%)$ & $2(10,52 \%)$ & $16(84,21 \%)$ \\
\hline 6 & Zero & Zero & Zero & $5(26,31 \%)$ & $14(73,68 \%)$ \\
\hline 7 & $2(10,52 \%)$ & Zero & $2(10,52 \%)$ & $5(26,31 \%)$ & $1(5,26 \%)$ \\
\hline 8 & $10(52,63 \%)$ & $2(10,52 \%)$ & $1(5,26 \%)$ & $5(26,31 \%)$ & $1(5,26 \%)$ \\
\hline 9 & $12(63,15 \%$ & $1(5,26 \%)$ & $1(5,26 \%)$ & $3(15,78 \%)$ & $2(10,52 \%)$ \\
\hline 10 & $13(68,42 \%)$ & $1(5,26 \%)$ & $2(10,52 \%)$ & $1(5,26 \%)$ & $2(10,52 \%)$ \\
\hline 11 & $7(36,84 \%)$ & Zero & $1(5,26 \%)$ & $4(21,05 \%)$ & $7(36,84 \%)$ \\
\hline 12 & $11(57,89 \%)$ & Zero & $3(15,78 \%)$ & $2(10,52 \%)$ & $3(15,78)$ \\
\hline 13 & $3(15,78 \%)$ & $1(5,26 \%)$ & $5(26,31 \%)$ & $6(31,57 \%)$ & $4(31,05 \%)$ \\
\hline 14 & $4(21,05 \%)$ & $1(5,26 \%)$ & $3(15,78 \%)$ & $8(42,10 \%)$ & $3(15,78 \%)$ \\
\hline
\end{tabular}

Fonte: Dados da pesquisa

TABELA 4 - Análise dos critérios e ações do fornecimento de hortifrutigranjeiros (pelo processo de licitação de compras)

\begin{tabular}{|c|c|c|c|c|c|}
\hline Número da Pergunta & $\begin{array}{c}\text { Discorda } \\
\text { totalmente }\end{array}$ & Discorda & $\begin{array}{c}\text { Imparcial/ } \\
\text { Neutro }\end{array}$ & Concorda & Concorda totalmente \\
\hline 1 & Zero & Zero & Zero & $1(9,10 \%)$ & $10(99,90 \%)$ \\
\hline 2 & Zero & $4(36,36 \%)$ & $1(9,10 \%)$ & $1(9,10 \%)$ & $5(45,45 \%)$ \\
\hline 3 & Zero & Zero & $1(9,10 \%)$ & $3(27,27 \%)$ & $7(63,63 \%)$ \\
\hline 4 & Zero & Zero & Zero & $1(9,10 \%)$ & $10(90,90 \%)$ \\
\hline 5 & $1(9,10 \%)$ & Zero & Zero & $2(18,18 \%)$ & $8(72,72 \%)$ \\
\hline 6 & $1(9,10 \%)$ & Zero & $1(9,10 \%)$ & $1(9,10 \%)$ & $8(72,72 \%)$ \\
\hline 7 & $3(27,27 \%)$ & Zero & Zero & $1(9,10 \%)$ & $8(72,72 \%)$ \\
\hline 8 & $3(27,27 \%)$ & $2(18,18 \%)$ & $1(9,10 \%)$ & $1(9,10 \%)$ & $4(36,36 \%)$ \\
\hline 9 & $5(45,45 \%)$ & $1(9,09 \%)$ & Zero & Zero & $5(45,45 \%)$ \\
\hline 10 & $3(27,27 \%)$ & $1(9,09 \%)$ & $1(9,10 \%)$ & Zero & $6(54,54 \%)$ \\
\hline 11 & $3(27,27 \%)$ & Zero & Zero & Zero & $8(64,64 \%)$ \\
\hline 12 & $6(54,54 \%)$ & $1(9,10 \%)$ & $1(9,10 \%)$ & Zero & $3(27,27 \%)$ \\
\hline 13 & Zero & $2(18,18 \%)$ & $5(45,45 \%)$ & Zero & $3(27,27 \%)$ \\
\hline 14 & Zero & $2(18,18 \%)$ & $3(27,27 \%)$ & $3(27,27 \%)$ & $3(27,27 \%)$ \\
\hline
\end{tabular}


Observou-se que, nos dois grupos de hospitais, o padrão do tamanho das frutas é uma característica fundamental no fornecimento, o que ainda é complementado por uma análise no recebimento das condições de forma, de formato e da cor dos hortifrutigranjeiros. Na visita técnica, observou-se que os gêneros são pré-selecionados de acordo com a necessidade do cliente. Porém, tanto para exclusão de gêneros impróprios para o consumo, como para os cuidados no manuseio dos produtos, apenas a metade dos fornecedores se detém ao cumprimento dessas questões.

Constatou-se, na pesquisa, no que diz respeito aos fornecedores, que o transporte não é adequado em $100 \%$ das amostras, não há controle de tempo e temperatura e as condições de armazenamento não seguem os padrões determinados. Foi informado pelos fornecedores que existem rotinas de higienização e limpeza de área física, caminhões, caixas, estrados e câmaras frias. Todavia, esta rotina não é registrada e varia de fornecedor para fornecedor, não havendo padronização formal. De acordo com as BPF, esses itens necessariamente precisam ser controlados [2, 19].

É importante a informação de que aproximadamente $60 \%$ dos hospitais devolvem mercadorias para os fornecedores quando as mesmas não se encontram em padrões previamente determinados. O destino dos produtos devolvidos, em $41,2 \%$, é o fornecimento deste produto a outro cliente, podendo ser outro hospital. Posteriormente, verificou-se que $26 \%$ dos fornecedores devolvem a mercadoria para o produtor. Esse fato demonstra a falta de comunicação e de coordenação entre os elos da cadeia de suprimentos [12].

A maioria (72\%) dos fornecedores não conhece a proveniência dos produtos e a existência de alguma certificação. A fase de pré-requisitos para a aquisição de gêneros tem extrema importância no serviço de nutrição. Se a matéria-prima não atender a especificações rigorosamente estabelecidas, compromete-se toda a qualidade definida como objetivo da Unidade de Alimentação e Nutrição (UAN). Deve-se adquirir gêneros somente de fornecedores que obedeçam rigorosamente às normas e determinações oficiais e que recebam inspeção dos órgãos competentes. É importante, também, realizar visitas periódicas às instalações do fornecedor para verificar a manutenção do padrão de qualidade [10, 11]. Porém, contatou-se que a maioria dos hospitais não realiza visitas técnicas antes de iniciar atividades com um novo fornecedor.

No fornecimento atual, observou-se que os fornecedores atendem diferentes clientes (restaurantes, supermercados, clínicas e hospitais), cada um deles com necessidades e exigências distintas, situação que não permite uma customização dos produtos pela diversidade de requisitos e falta de conhecimento por parte dos fornecedores.

Os hospitais consideram necessário que os hortifrutigranjeiros sejam isentos de resíduos químicos e que existam controles na utilização de agrotóxicos. No entanto, aproximadamente $45 \%$ dos hospitais não realizam análises químicas ou microbiológicas nos hortifrutigranjeiros, para averiguar a ausência de substâncias deletérias.

\section{4 - CONCLUSÕES}

Os resultados obtidos permitem concluir que o processo de fornecimento de hortifrutigranjeiros aos hospitais não é eficiente, uma vez que as normas técnicas não são seguidas por parte dos fornecedores. Quanto aos hospitais, o recebimento não é adequado, e em geral o profissional que recebe as mercadorias não tem conhecimento técnico, nem tampouco segue a legislação vigente para este procedimento.

No que se refere à utilização de agrotóxicos e isenção de resíduos, a questão parece ser mais preocupante. Os fornecedores não conhecem a proveniência dos produtos, não sabem se existe certificação e a maioria desconhece a existência de controle na utilização de agrotóxicos. Os hospitais não conhecem a proveniência dos produtos e a grande maioria não realiza análises para verificar a segurança dos alimentos.

Sugere-se a implementação da metodologia de Análise dos Perigos e Pontos Críticos de Controle, das Boas Práticas de Fabricação, do Gerenciamento da Cadeia de Suprimentos e do Cooperativismo, como propostas para a otimização do fornecimento.

A inexistência de um sistema efetivo de exigência sobre direitos e sobre premissas de saúde e segurança dos alimentos por parte dos hospitais, somada à baixa ação fiscal (sanitária e tributária) faz com que a qualificação do setor dos hortifrutigranjeiros tenha predomínio na informalidade, com baixo padrão de qualidade. A estratégia de mudança proposta deve estar ancorada em informação ao consumidor e aos produtores, com foco em problemas e alternativas, em fiscalização com direcionamento em saúde pública e na divulgação das ações.

As constatações deste estudo mostram que o processo atual compromete a saúde e a qualidade de vida dos que recebem a alimentação hospitalar. Os danos, muitas vezes, não se mostram de imediato, porém podem ter efeito cumulativo e apresentarem-se posteriormente à internação hospitalar.

\section{5 - REFERÊNCIAS BIBLIOGRÁFICAS}

[1] ARRUDA, G.A. Método APPCC - Opinião. Disponível em: http://www.nutrinews.com.br/serviços/opinião/ appcc;html. Acesso em: 12 ago. 2002.

[2] ARRUDA, G.A.; POPOLIM, W.D.; FUJINO, H.; LEITE, C.L.; RIBEIRO, L.C. Avaliação das condições de entrega de gêneros perecíveis em unidades de alimentação e nutrição, através do método de análise de perigos em pontos críticos de controle (APPCC). Higiene alimentar, v. 10, n. 44, p. 44-48, 1996.

[3] BRASIL. Ministério da Saúde. Portaria n ${ }^{\circ} 1.428 / \mathrm{MS}$, de 26 de novembro de 1993. Aprova o regulamento técnico para inspeção sanitária de alimentos, as diretrizes para o estabelecimento de boas práticas de produção e de prestação de serviços na área de alimentos e o regulamento técnico para o estabelecimento de padrões de identidade e qualidade (PIQ's) para serviços e produtos na área de alimentos. Disponível em: http://anvsl.saude. gov.br/Procuradoriaalimentos/\%20portaria\%201428_ 93.html Acesso: em 5 fev. 2000. 
[4] BRASIL. Ministério da Saúde, Secretaria de Vigilância Sanitária. Portaria no $326-S V S / M S$, de 30 de julho de 1997. Aprova o regulamento técnico; condições higiênicos-sanitárias e de boas práticas de fabricação para estabelecimentos produtores/industrializadores e de alimentos. Disponível em: http://www.anvisa.gov.br/legis/portarias/326_97.htm. Acesso em: 24 ago. 2000.

[5] BRASIL. Agência Nacional de Vigilância Sanitária. Resolução ANVS nº18, de 18 de novembro de 1999. Republica a Resolução 363, de 29 de julho de 1999. Disponível em: http://www.anvisa.gov.br/legis/resol/18_ 99_anvs.htm. Acesso em: 24 ago. de 2000.

[6] BRYAN, F.L. Prevention of foodborne diseases in food service establishments. Journal of Environmental Health, v. 41, n. 4, p. 198-206, 1979.

[7] CHRISTOPHER, M. Logística e gerenciamento da cadeia de suprimentos: estratégias para a redução de custos e melhoria dos serviços. São Paulo: Pioneira, 1997.

[8] COOPER, M.C.; ELLRAM, L.M. Characteristic of supply chain management and implications for purchasing and logistics strategy. The International Journal of Logistics Management, v. 4, n. 2, 1993.

[9] DAMBORIARENA, E. Certificação e rotulagem como fator de competitividade na cadeia dos hortigranjeiros no Estado do Rio Grande do Sul. 2001.129 p. Dissertação (mestrado em Administração) - Universidade Federal do Rio Grande do Sul.

[10] DAVIS, T. Effective supply chain management. Sloan Management Review, Summer, 1993.

[11] FERNANDES, A.T.; FERNANDES, M.O.V.; RIBEIRO FILHO, $\mathrm{N}$. Infecção hospitalar e suas interfaces com a área da saúde. São Paulo: Atheneu, 2000. p. 1208-1218.

[12] FERNANDES, O.A.; BUENO, A.F. MIP Receituário agronômico, 2002.

[13] GREIS, N.P.; KASARDA, J.D. Enterprise logistics in the information era. California Management Review, v. 39, n. 4, 1997.
[14] INMETRO. Site. Disponível em: http://www.inmetro. gov.br. Acesso em: ago 2002.

[15] LEE, H.L.; BILLINGTON, C. Managing supply chain inventory: pitfalls and opportunities. Sloan Management Review, v. 33, n. 3, 1992.

[16] LEWIS, R.G.; VOEHL, F.; STEIN, M. Macro logistics: supply chain management. Paper presented at the eighth Annual Conference of the Production and Operations Management Society, Miami BeachFLA, Apr. 1997.

[17] QUEIROZ, A.T.A.; RODRIGUES, C.R.; ALVEZ, G.G.; KAKISAKA, L.T. Boas práticas de fabricação em restaurantes self-service a quilo. Higiene Alimentar, v. 14, n. 78/79, p. 45-49, 2000.

[18] RODRIGUES, H.R. Manual de rotulagem. Rio de Janeiro: Embrapa, 1999.

[19] SÃO PAULO. Secretaria de Estado da Saúde. Resolução SSE/SUS-196, de 29 de dezembro de 1998. Roteiros e guias de inspeção em Vigilância Sanitária: ficha de inspeção de estabelecimentos na área de alimentos. Disponível em: http://www.saude.sp.gov.br/html/fr_links. htm. Acesso em: 25 ago. 2000.

[20] SBCTA - SOCIEDADE BRASILEIRA DE CIÊNCIA E TECNOlOGIA DE Alimentos. Programa de fornecimento com garantia de qualidade para as empresas de alimentos. $3^{\mathrm{a}}$ ed. Campinas: Profíqua, 38 p., 1996.

[21] SILVA JR, E.A. Manual de controle higiênicosanitário em alimentos. $2^{\mathrm{a}}$ ed. São Paulo: Varela, 385 p., 1997.

[22] WOOD, T. Jr.; ZUFFO, P.K. Supply Chain Management. Revista de Administração de Empresas, v. 38, n. 3, 1998.

[23] ZYLBERSZTAJN, D; NEVES, M.F. Economia e gestão dos negócios agroalimentares. Pensa. São Paulo: Pioneira, 2000. 